\title{
Welcome to Toronto. Welcome to the CIHR
}

\author{
Malcolm King, PhD, President, Canadian Thoracic Society
}

$\mathrm{M}$ ay 6 to 10, 2000 is a great time for the Canadian respiratory community. It is the 100th anniversary of our Lung Association, and it is our chance to host the respiratory world at the American Lung Association, American Thoracic Society International Conference and the Canadian Thoracic Society Annual Meeting.

The name Toronto comes from a Mohawk word meaning 'meeting place'. The rivers that run into Lake Ontario were traditional gathering places for the aboriginal peoples that lived in this area before the coming of the European settlers. My ancestors include both Mohawks and the Mississaugas of the Credit, the native tribe that occupied the Toronto area as its traditional territory until the late 18 th century.

As we begin this new century, Toronto has certainly become Canada's international meeting place. Enjoy the city. Enjoy an exciting conference. And in the spirit of the ancestors, welcome.

This month represents another opportunity for welcome: Welcome to our new Canadian Institutes of Health Research (CIHR), and farewell to the Medical Research Council (MRC) of Canada. While writing this editorial, Bill 13 to establish the CIHR had passed through the House of Commons with a clear majority and was awaiting approval by the Senate, normally a formality but never to be taken lightly. Some CIHR funding programs are already underway, and our health research community is anxious to see how the whole structure will take shape.

Our American visitors will recognize the many similarities to the long-established National Institutes of Health (NIH). Indeed, many of the features (but not all) of our new medical research structure are patterned after the NIH, most notably a dozen or so institutes based on thematic groupings such as cancer, genetics or infection. The details remain to be decided, and are anxiously awaited by the Canadian research community.

The experiences of a number of other countries were also drawn upon, and it is hoped the CIHR, as a synthesis of this collective experience, will be an improvement over existing models. The other big difference, continuing with established MRC practice, is that health research will continue to be conducted in universities and health centres, and not in central laboratories, as is the case with the NIH complex. In fact, because this is the 21 st century and the

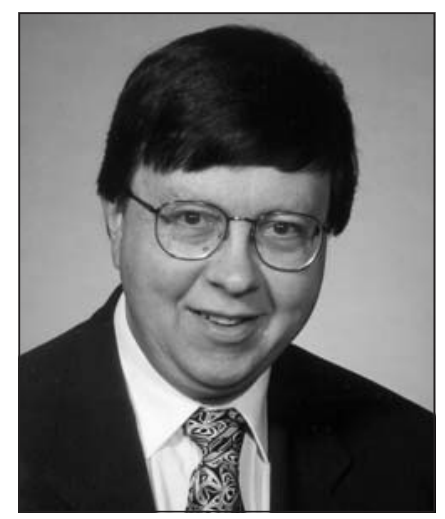
electronic age, CIHR research units will be virtual in nature, as will most of the CIHR budget. That is to say, there will be real money for the front-line investigators - a substantial increase from the MRC days, in fact - but for each institute, the majority of its funding will be virtual because most grants will be awarded directly to the investigators by the CIHR central administration and not by the institiutes themselves. Still, there will be real advantages associated with each institute, since they will have a chance to establish priorities and new directions for research, and their thematic structure should allow for better opportunities to foster interaction between scientists with common interests.

The beginning of the CIHR also is a time of uncertainty for many researchers in this country. The main uncertainty, even anxiety, is the sense of not knowing where one's research will fit into the overall plan. Instead of research being grouped in the traditional MRC peer-review committees, it will be assigned to large, as yet-to-be designated thematic institutes. The respiratory research community is facing this problem, and it is a cause for great concern. Where will respiratory research fit in? It seems that our first choice of having a dedicated respiratory and critical care health research institute has probably been rejected, and our community is faced with the likelihood that a much larger institute, grouping 


\section{President's page}

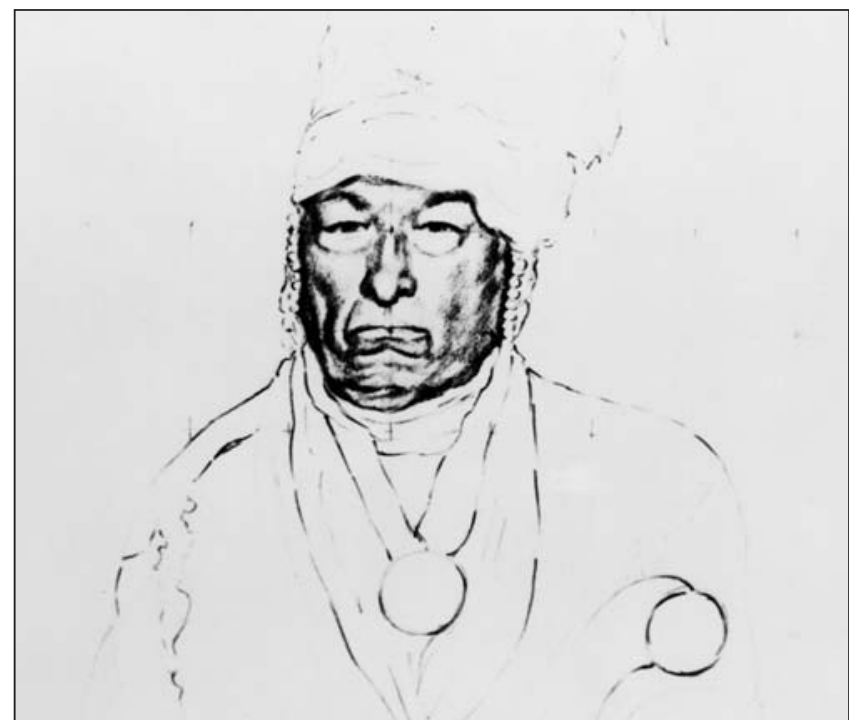

Figure 1) Chief George King, last of the traditional chiefs of the Mississaugas of the Credit First Nation, was the author's greatgreat-grandfather. He was born on Toronto Island in 1813 and died at New Credit, Ontario in 1871 respiratory research with cardiovascular and cerebrovascular themes, is the most likely scenario. As it stands now, however, we are in limbo; nothing has been anounced, and nothing is certain. Our community has been severely divided and discouraged by this issue.

The respiratory research component of the CIHR may, thus, end up as a minority partner in a cardio-cerebrovascular respiratory institute along the lines of the United States National Heart, Lung and Blood Institute (NHLBI). If this turns out to be the case, perhaps we can take some comfort from the NIH model. In the United States, the Division of Lung Diseases of the NHLBI has served the academic respiratory community well, and respiratory health research has had many opportunities to move forward within this divisionalized structure. Given the likelihood of a similar grouping in our CIHR, our community will have to adapt and negotiate for the best arrangement to protect the interests of lung health research and allow its agenda to move forward. There is an advertisement on many airline magazines that says "You only get what you negotiate."The time has come, the walrus said, to begin the negotiations. 


\section{Bienvenue à Toronto. Bienvenue aux ICRS}

\section{Malcolm King, PhD, Président de la Société Canadienne de thoracologie}

L a communauté canadienne de pneumologie aura le cœur à la fête du 6 au 10 mai 2000. Ce sera le $100^{\mathrm{e}}$ anniversaire de l'Association pulmonaire du Canada et nous aurons l'honneur d'accueillir, en l'occasion, des hôtes à la conférence internationale de l'American Lung Association et de l'American Thoracic Society et au congrès annuel de la Société canadienne de thoracologie.

Le nom de la ville de Toronto vient d'un mot mohawk qui veut dire « lieu de rencontre ». Les rivières qui se jettent dans le lac Ontario étaient le lieu traditionnel de rencontre des peuples autochtones qui vivaient dans la région avant l'arrivée des colons européens. Parmi mes ancêtres figurent des Mohawks et des Mississaugas of the Credit, la tribu qui occupait la région de Toronto, son territoire traditionnel, jusqu'à la fin du XVIIIe siècle.

Au tournant du millénaire, Toronto est devenue, sans doute, le lieu de rencontre international du Canada. Alors, découvrez la ville! Assistez à une conférence des plus intéressantes! Et, dans le même esprit d'accueil de mes ancêtres, je vous dis «Bienvenue! »

Avril est aussi l'occasion d'accueillir une autre nouveauté : les Instituts canadiens de recherche en santé (ICRS), mais, du même souffle, il doit faire ses adieux au Conseil de la recherche médicale $(\mathrm{CRM})$ du Canada. Pendant que je rédigeais le présent éditorial, la Chambre des communes a voté, avec une forte majorité, en faveur du projet de loi 13, constituant les ICRS, et celui-ci doit être approuvé par le Sénat; ce devrait, en principe, n'être que formalité, mais il ne faut rien tenir pour acquis. Certains programmes de financement des ICRS ont déjà été mis sur pied, et le monde de la recherche en santé a bien hâte de voir quelle forme prendra toute la structure.

Nos hôtes américains reconnaîtront certainement les nombreuses similitudes entre les ICRS et les National Institutes of Health (NIH) établis depuis longtemps. En effet, on s'est inspiré fortement, mais pas totalement, des NIH pour ériger la nouvelle structure de la recherche en santé, l'élément le plus digne de mention étant la constitution d'une dizaine d'instituts fondés sur de grands thèmes comme le cancer, la génétique, les infections. Il reste à en définir les détails, et la communauté de chercheurs au Canada attend avec impatience le résultat final.

Les ICRS sont également le fruit d'expériences vécues dans d'autres pays, et, de ce fait, il est à espérer qu'ils seront une version améliorée des modèles actuels. Une différence importante à signaler est le fait que la recherche se fera, comme c'est le cas actuellement avec le CRM, dans les universités et les centres hospitaliers, et non dans des laboratoires centraux, comme c'est le cas avec les

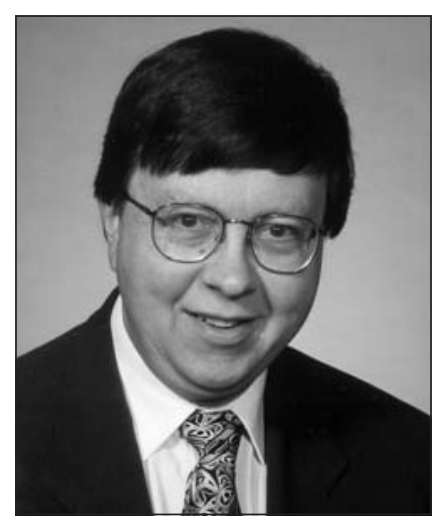
NIH. Voyant le jour au début du XXI ${ }^{\mathrm{e}}$ siècle et à l'ère électronique, les ICRS seront des unités de recherche virtuelles, tout comme leur budget d'ailleurs. Cela veut dire qu'il y aura certes de l'argent consacré à la recherche - et les fonds seront sensiblement augmentés par rapport à ceux que reçoit le CMR -, mais il sera versé directement aux chercheurs de première ligne par l'administration centrale des ICRS et non par les instituts eux-mêmes. C'est pourquoi la plus grande partie du financement des ICRS sera virtuel. Par ailleurs, les instituts y trouveront leur compte; ils pourront, par exemple, établir leurs priorités et donner de nouvelles orientations à la recherche, et leur structure thématique devrait favoriser les échanges entre les scientifiques qui partagent des points d'intérêt communs.

Toutefois, l'arrivée des ICRS ne se fait pas sans incertitude, voire anxiété, pour bien des chercheurs au Canada parce qu'ils ne savent pas comment leur recherche cadrera dans le plan général. Au lieu d'être groupée dans les comités traditionnels de révision par les pairs du CRM, la recherche sera confiée à de gros instituts, dont la vocation thématique n'a pas encore été désignée. La communauté de pneumologie doit faire face au problème, et cela est source de grande préoccupation. Quel sort attend la recherche en pneumologie? Il semble bien que notre premier choix d'avoir notre propre institut de recherche, consacré aux soins respiratoires et intensifs, a été rejeté et que notre communauté sera intégrée à un institut beaucoup plus gros, regroupant les maladies cardio-vasculaires, les accidents vasculaires cérébraux et la recherche en pneumologie. Mais, pour le moment, nous ne savons pas où nous allons aboutir; rien n'a été annoncé, rien 


\section{La page du président}

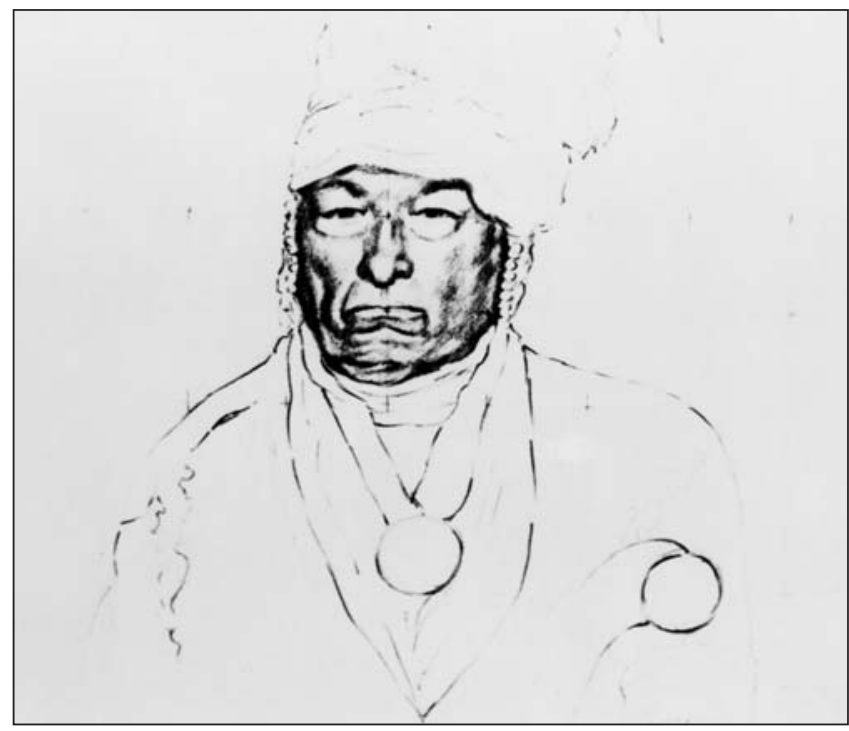

Figure 1) Le chef George King, le dernier des chefs traditionnels de la tribu des Mississaugas of the Credit First Nation, est l'arrièrearrière-grand-père de l'auteur. Il est né sur la Toronto Island en 1813 et est mort à New Credit, en Ontario, en 1871 n'est certain. Notre communauté se trouve profondément déchirée par la question et cela draine beaucoup d'énergie.

Il se pourrait fort bien que le volet de la recherche en pneumologie devienne un partenaire minoritaire dans un institut consacré aux maladies cardio-vasculaires, vasculaires cérébrales et respiratoires, suivant le modèle américain du National Heart, Lung and Blood Institute (NHLBI). Si tel est le cas, peut-être pouvons-nous nous consoler devant le succès que connaît le modèle des NIH. En effet, la division des maladies pulmonaires du NHLBI, aux États-Unis, a bien servi la communauté universitaire en pneumologie, et la recherche dans le domaine a su très bien tirer son épingle du jeu dans cette structure divisée. Comme il est fort probable que notre ICRS sera regroupé de la même façon, notre communauté aura à s'adapter et à négocier les meilleurs arrangements possible pour protéger ses intérêts en ce qui concerne la recherche en santé pulmonaire et pour maintenir le cap. Un message publicitaire parait souvent dans les revues offertes dans les avions; il dit, en substance, que l'on n'obtient que ce que l'on négocie. En bien, le temps est venu, comme on dit, de négocier. 


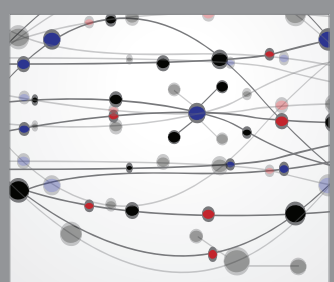

The Scientific World Journal
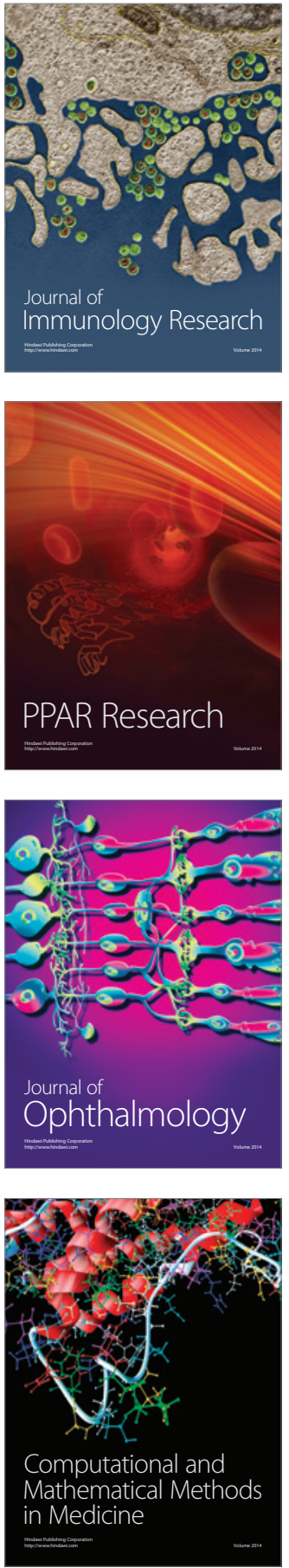

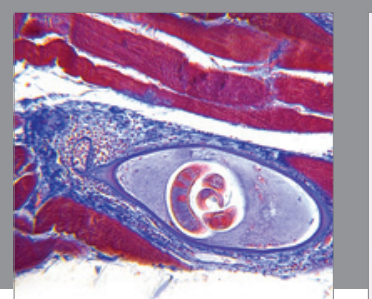

Gastroenterology Research and Practice

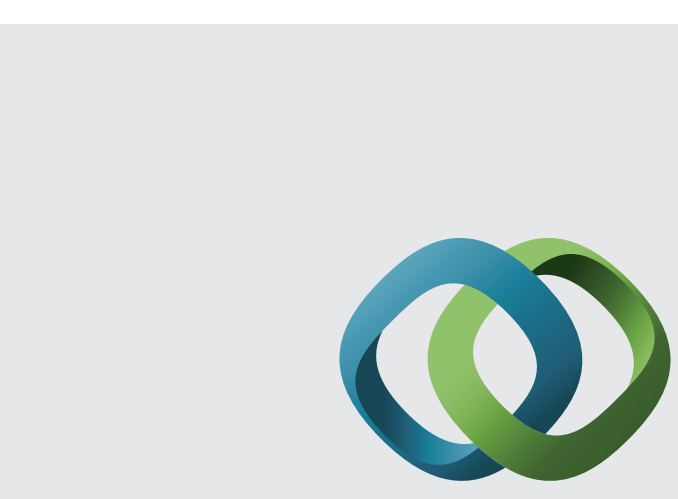

\section{Hindawi}

Submit your manuscripts at

http://www.hindawi.com
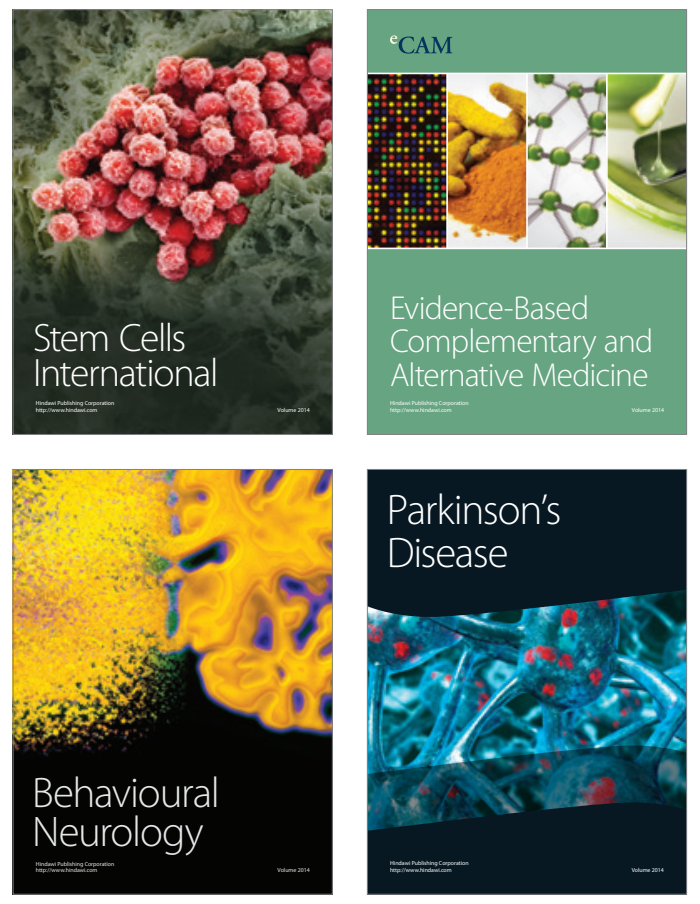
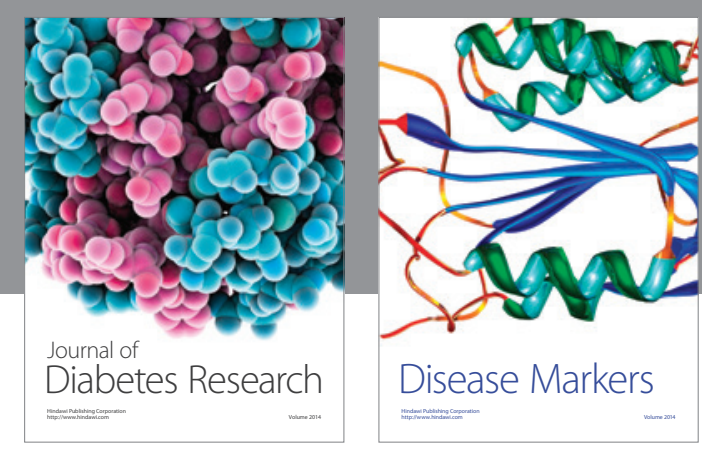

Disease Markers
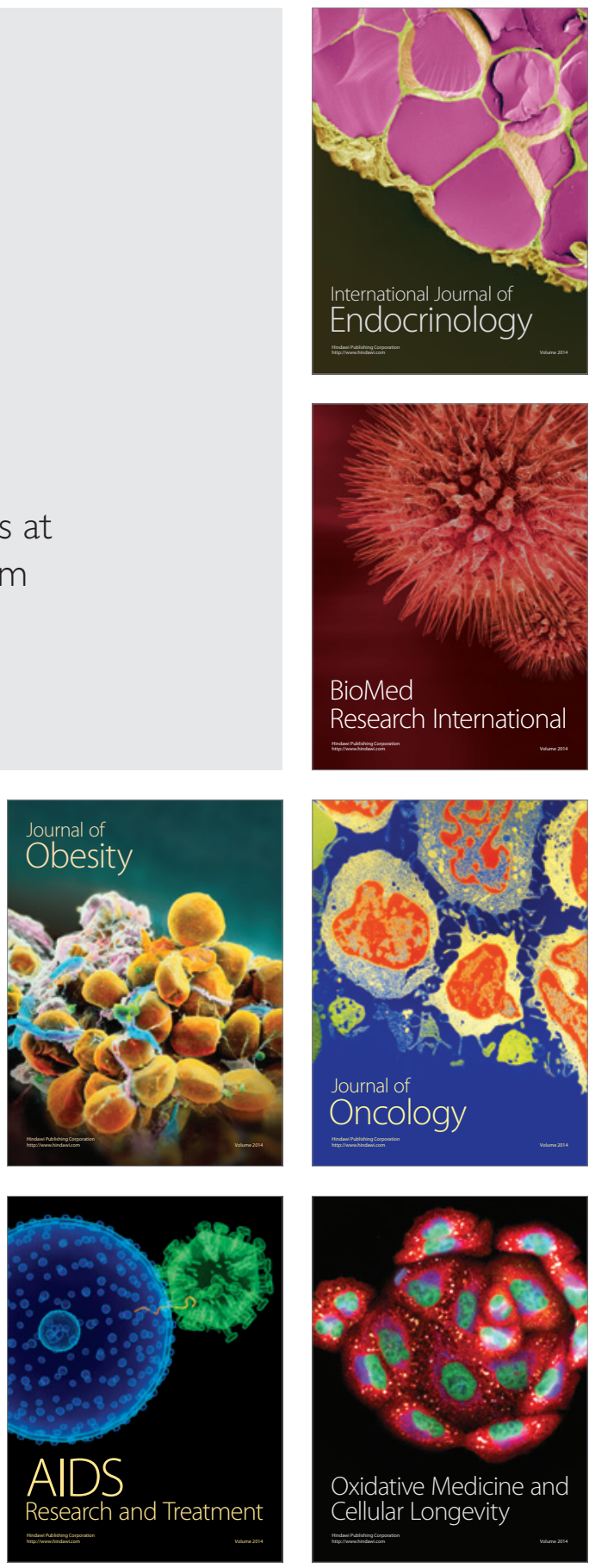